\title{
Nanoindentation of Silicate Low-K Dielectric Thin Films
}

\author{
Joseph B. Vella ${ }^{1}$, Alex A. Volinsky ${ }^{1}$, Indira S. Adhihetty ${ }^{1}$, N.V. Edwards, and William \\ W. Gerberich ${ }^{2}$ \\ ${ }^{1}$ Motorola, Digital DNA ${ }^{\mathrm{TM}}$ Labs, Process and Materials Characterization Lab, Mesa, AZ. \\ ${ }^{2}$ University of Minnesota, Dept. of Chem. Eng. and Mat. Science, Minneapolis, MN.
}

\begin{abstract}
The capabilities of nanoindentation to characterize low-k organo silicate glass (OSG) thin films is explored as a relatively rapid and inexpensive metric of mechanical properties, adhesion strength, and fracture toughness. One method of decreasing the static dielectric constant of OSG interlayer dielectrics requires the introduction of porosity in the material which has a dramatic impact on its mechanical and toughness properties. Percolation theory is used to formulate a correlation between porosity and elastic modulus. Using cube corner diamond indentation and scratch testing fracture toughness calculations are also discussed.
\end{abstract}

\section{INTRODUCTION}

A viable low-K material must be compatible with dual-damascene lithography, as well etching, stripping and cleaning processes--especially CMP and device packaging methods. The reliability of devices containing these multi-layer dual-damascene stacks depends on several factors, including: device mechanical stability; adhesion of the barrier metal to the low-K film; adhesion of etch stop, hard mask and capping layers to the low-K film and barriers; and the ability to polish the $\mathrm{Cu}$ and package device without pattern shift. Since the dual-damascene stack is exposed to high shear stresses during the CMP process, any flaws at the interfaces or in the low-K film itself can lead to long-term reliability problems. Given that low-k OSG materials gain their advantageous dielectric properties by the introduction of lattice defects in the form of pores, detailed reliability and compatibility tests are required to integrate new low-K dielectric materials and $\mathrm{Cu}$ interconnects.

Mechanical properties of thin films often differ from those of the bulk materials. Due to typically high yield strengths thin films can support very high residual stresses. This residual stress can be relieved later during processing or in the actual device operation through thin film fracture, or interfacial delamination. The most important properties of low-k materials necessary to insure device reliability are elastic modulus, hardness, interfacial adhesion, and film fracture toughness.

\section{EXPERIMENT}

Several test structures have been constructed to test OSG low-k dielectric films mechanical properties. OSG films of different thicknesses ranging from $50 \mathrm{~nm}$ to 2.5 microns were deposited on oxidized Si wafers (with a $50 \mathrm{~nm}$ sputtered TaN glue layer) using a precursor CVD process. Thin film mechanical properties can be measured by tensile testing of freestanding films [1] and by the microbeam cantilever deflection technique [2, 3], but the easiest way is by nanoindentation, where no special sample preparation is required and tests can be performed 
quickly and inexpensively. During the measurement a sharp diamond indenter is forced into the tested material while continuously recording both the force and the indentation depth. Mechanical properties are measured by either analyzing the continuous load-displacement profile or by measuring the material response to a frequency modulated force oscillation. Both elastic modulus and hardness can be readily extracted from the load versus displacement curve [4-6]. Since the depth resolution is on the order of nanometers, it is possible to indent even very thin $(100 \mathrm{~nm})$ films. Indentation has been also used to measure thin film adhesion [7-12], where the mechanical energy release rate, or practical work of adhesion is calculated based on the delamination size. Similar fracture properties such as fracture toughness or adhesion strength are derived from the continuous load-displacement profile and an independently measured geometrical scale parameter such as crack length or delamination radius.

Nanoindentation techniques are being explored for measuring thin film fracture toughness. When a sharp tip such as Vickers, Berkovich or a cube corner diamond is indented into bulk brittle materials, radial cracking usually occurs after a critical load has been reached, which allows ones to calculate fracture toughness based on the maximum indentation load and the crack length [16-18]. This method of analysis in complicated in the case of thin film radial fracture because of the half penny crack shape perturbation by the substrate, film densification, and residual stresses in the film. Current studies have yielded promising developments in this area however.

\section{RESULTS AND DISCUSSION}

During the qualification of a CVD tool for OSG low-k material deposition, nanoindentation was used as a metric for the films. Figure 1a shows the load displacement profiles of the baseline film as well as two of the tool transfer films. Decreased hardness and modulus in the tool transfer films is clear from the decreased loading and unloading slopes. It is also interesting to note that these films did not exhibit any appreciable plasticity despite greater than $10 \%$ of the film thickness being indented. Spectroscopic ellipsometry performed on these films showed a substantial difference in refractive index, which given chemical similarity, suggest difference in relative electron density or porosity (Fig. 1b) $[19,20]$.
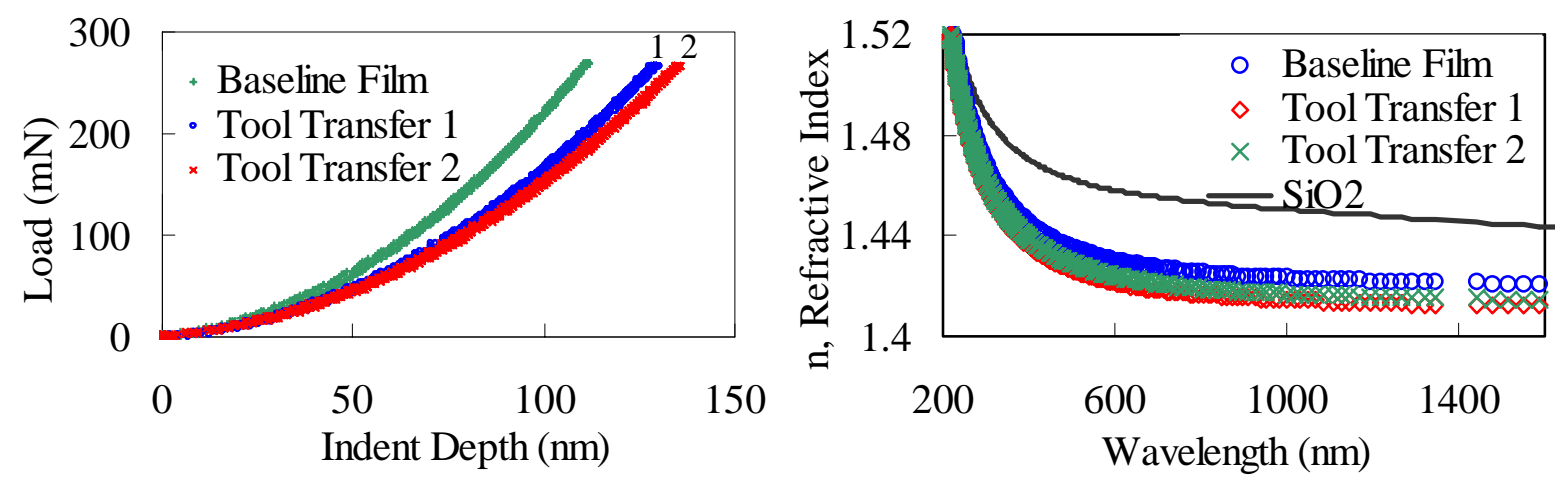

Figure 1. a) Load-displacement curves: note that these also represent unloading curves for $2 \mu \mathrm{m}$ thick low-K films, b) Refractive index for the baseline and tool transfer films. 
While exploring the process space of this new tool, including reaction temperature, partial pressures of inert and reactant gases, films of varying mechanical properties were generated. In the absence of dramatic chemical changes in the films across these process changes, the porosity may be monitored quantitatively if its affect on mechanical properties can be effectively mapped. One promising method of doing this is by using percolation theory, using the empirical relation:

$$
\frac{E^{*}}{E_{0}}=\left(1-\frac{p^{*}}{p_{c}}\right)^{f}
$$

where $\mathrm{E}^{*}$ is the elastic modulus of the material under study, $\mathrm{E}_{0}$ the elastic modulus of the ideal, fully dense material, $p$ the porosity of the material under study, $p_{c}$ the percolation threshold at which the elastic modulus goes to zero [12]. In this way, mechanical properties measurements can be used as a rapid and inexpensive porosity metric. Other mechanical and fracture properties can be used as well [13]. Low-k materials in particular have been deposited to extremely high porosities $(<95 \%)$ such as seen in aerogels, therefore it could be argued that it is reasonable to assume $p_{c}$ to be at or near $100 \%$ porosity [14]. Assuming $E_{0}$ is $72 \mathrm{GPa}$ for the fused silica with zero porosity, and some independently verified measurements of OSG porosity, a plot similar to Fig. 2 can be generated. Here, the percolation exponent $f$ is 5.1. The elastic modulus was chosen as the porosity metric because as can be seen in Fig. 1a, the nanoindentation contacts are purely elastic. Therefore measures of hardness are merely second measures of the materials elastic properties. In fact, a linear correlation is seen between modulus and hardness in Fig. 3.

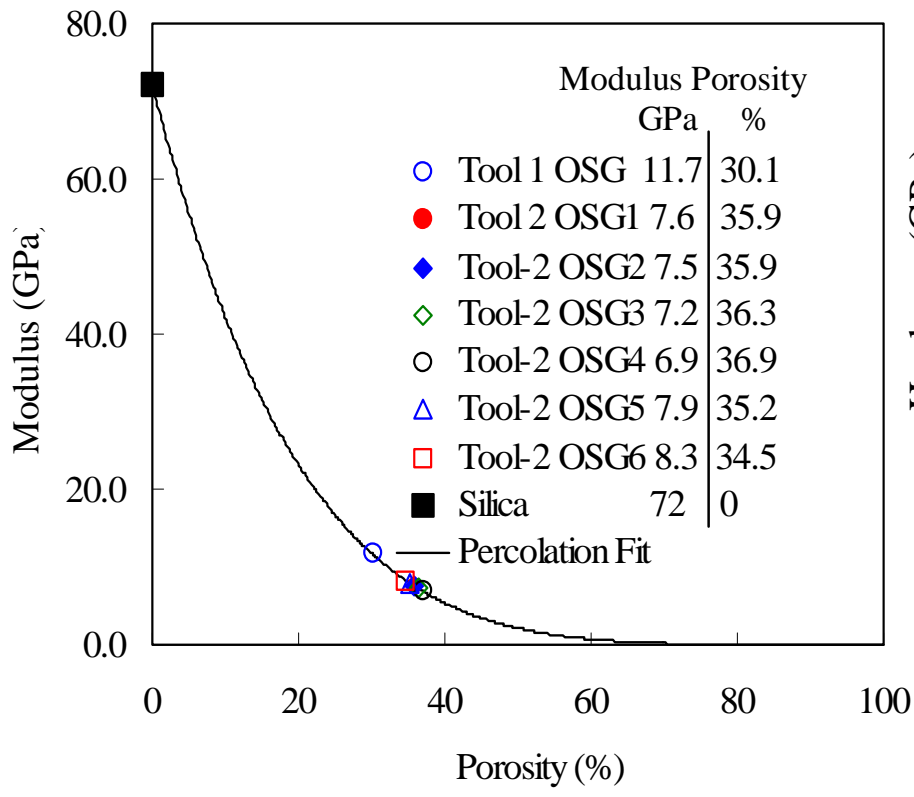

\section{Figure 2. Elastic modulus as a function of porosity.}

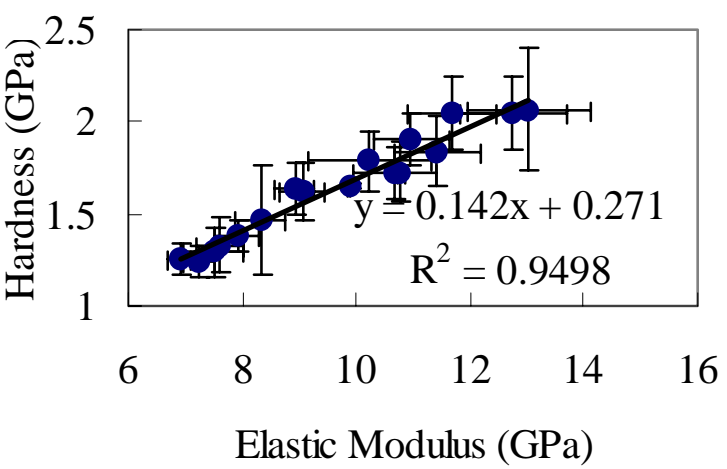

Figure 3. Linear plot of a low-k dielectric film hardness versus modulus, demonstrating the interrelated mechanical properties that stem largely from their porosity.

Beyond measuring the mechanical properties significant advances have been made recently in measuring the adhesion strength of thin films using nanoindentaiton. With the high hardness to modulus ratios one may expect low fracture toughness and adhesion of these 
materials. Adhesion of low-K dielectric films was measured by means of a superlayer indentation technique [9-12]. Most well-adhered or low modulus thin films can not be delaminated by means of regular indentation: films would rather deform plastically around the indenter by forming pileup, or they would not be able to carry the indentation stress to the crack tip. To prevent these problems a high modulus hard superlayer, capable of supporting and storing large amounts of elastic energy is deposited on top of the film of interest. Upon indentation a delamination blister forms around the indent, and its area is used to calculate the strain energy release rate (practical work of adhesion).

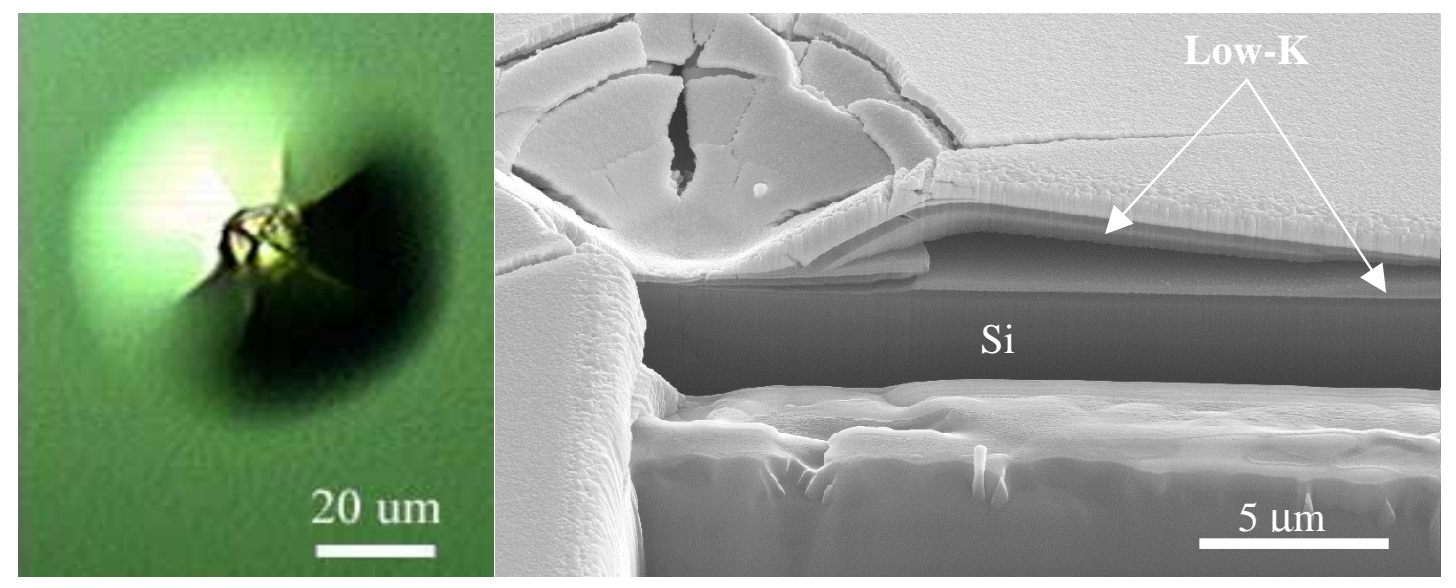

Figure 4. Typical indentation-induced blister and its FIB cross-section.

Several delamination blisters have been cross-sectioned using Focused Ion Beam (FIB), and it was found that the low-K fracture is cohesive (Fig. 4), so what is really measured is the fracture toughness of the low-K film, not the interfacial adhesion.

After realizing that in most cases there is a competition between the interfacial adhesion and film toughness, and that cohesive fracture is observed in the case of low-K films, we made attempts to measure thin film toughness more accurately using cube corner nanoindentation. Fracture toughness of a bulk brittle material can be calculated within $40 \%$ accuracy based on the maximum indentation depth, $P_{\max }$ and the crack length, $c[17,18]$ :

$$
K_{C}=\alpha\left(\frac{E}{H}\right)^{1 / 2}\left(\frac{P_{\max }}{c^{3 / 2}}\right)
$$

where $\alpha$ is an empirical constant which depends on the geometry of the indenter, and is 0.0319 for a cube corner indenter geometry [17], $E$ is the elastic modulus, and $H$ is the mean hardness. This expression can not be directly applied in the case of a thin film, since typically the crack shape is no longer halfpenny shape anymore. It was also noted that the maximum indentation load scales linearly with the crack length to the $3 / 2$ power, so as a first order approximation equation (2) can be used to estimate low-K films fracture toughness [18]. Using this method we estimate low-K dielectric films fracture toughness to range from 0.01 to $0.05 \mathrm{MPa} \cdot \mathrm{m}^{1 / 2}$. Figure 5 shows the indentation-induced radial crack in the low-K film. It was interesting to note that these cracks propagated under the electron beam in SEM. 

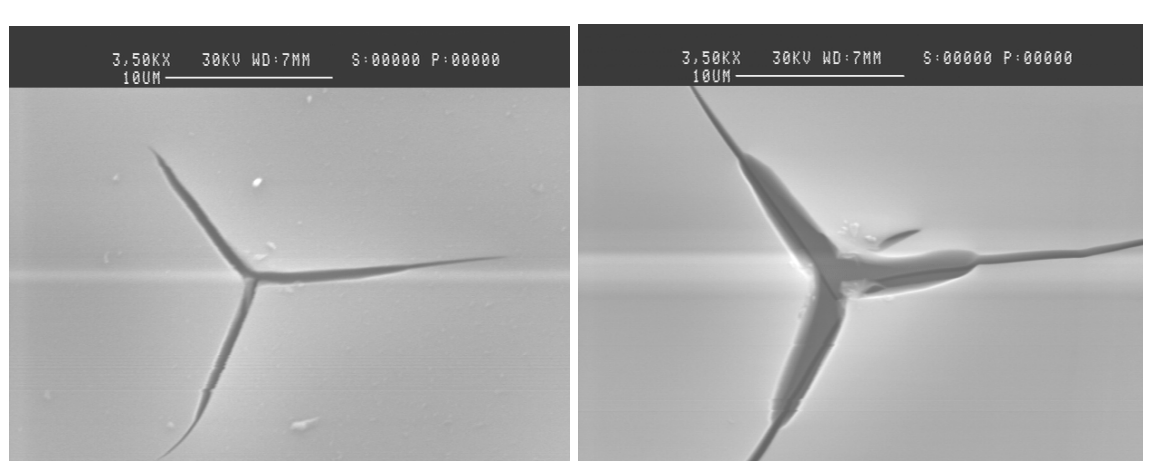

Figure 5. Indentationinduced low-K film fracture.

An additional method utilized for measuring toughness involves a lateral scratch, which causes a tangential stress at the trailing edge of the scribe. This has been utilized by both Ostartage, et al [22] and Hoehn, et al [23], noting that

$$
K_{C}=2 \sigma_{\theta \theta}\left(\frac{c}{\pi}\right)^{1 / 2} \cdot \sin ^{-1}\left(\frac{a}{c}\right)
$$

where $a$ is the contact radius and $c$ is the half crack. Since $a / c$ is almost always less than $1 / 2$, then $\sin ^{-1}(\mathrm{x}) \sim \mathrm{x}$ and with $\sigma_{\theta \theta}=P_{\max } / \pi a^{2}$, one finds that equation 3 reduces to:

$$
K_{C} \approx \frac{2 P_{\max }}{\pi^{3 / 2}} \cdot \frac{1}{a c^{1 / 2}} \approx \text { const } \cdot\left(\frac{P_{\max }}{c^{3 / 2}}\right)
$$

with the latter approximation coming if $c / a \sim$ constant. This then is the same as equation (2), since $(E / H)^{1 / 2}$ is nearly constant in Figure 3. However, both equation (2) and (4) have inherent composite yield strength, modulus and strain energy release rate built into a laminate system needing detailed analysis. One of the ways to more accurately solve this problem would be to use FEM calculations of the stress field around the indenter, taking into account low-K film thickness and residual stress.

For several low-K materials there is a critical thickness of approximately $3 \mu \mathrm{m}$ at which the film fractures due to the residual stress relief. This allowed us to estimate an upper bound of $0.06 \mathrm{MPa} \cdot \mathrm{m}^{1 / 2}$ for low-K film fracture toughness, taking $25 \mathrm{MPa}$ tensile residual stress for a 3 $\mu \mathrm{m}$ thick film [18]. A failure criterion based on the thin film residual stress is also proposed in [18].

Since fracture toughness strongly depends on the flaw size, preliminary measurements of low-k relative electron density/ porosity with spectroscopic ellipsometry have been made [20], but at this point more thorough theoretical analysis is required to accurately assess thin film fracture toughness using the nanoindentation technique in order to correlate the two types of measurements. 


\section{CONCLUSIONS}

As nanotechnology devices shrink in size, there are opportunities that low-k dielectrics offer. In pursuing there, there are significant mechanical weaknesses inherent to these materials that must be improved. Here, analytical techniques that utilize nanoindentation to measure low-k film hardness, modulus, interfacial adhesion, and fracture toughness as affected by thin film porosity, are the metrics being used in establishing this goal.

\section{REFERENCES}

1. D. T. Read, J.W. Dally, J. Mater. Res., 8(7), pp. 1542-1549 (1993).

2. T.P. Weihs, S. Hong, J.C. Bravman, and W.D. Nix, J. Mater. Res. 3(5), pp. 931-942 (1998).

3. S.P. Baker and W.D. Nix, J. Mater. Res. 9(12), pp. 3131-3152 (1994).

4. M. Doerner and W.D. Nix, J. Mater. Res. 1, p. 601 (1986).

5. G.M. Pharr, W.C. Oliver, F. Brotzen, J. Mater. Res., 7 (3), pp. 613-617, (1992)

6. W.C. Oliver and G.M. Pharr, J. Mater. Res., 7, pp.1564-1583, (1992)

7. D.B. Marshall and A.G. Evans, , J. Appl. Phys., 56, 2632, (1984)

8. J.J Vlassak, M.D. Drory and W.D. Nix, J. Mater. Res., Vol. 12, No. 7, p. 1900, (1997)

9. M.D. Kriese and W.W. Gerberich, J. Mater. Res., 14 (7), 3007, (1999)

10. M.D. Kriese, W.W Gerberich, N.R. Moody, J. Mater. Res., 14 (7), 3019, (1999)

11. A.A Volinsky, N.I. Tymiak, M.D Kriese, W.W Gerberich and J.W. Hutchinson, Materials Research Society Symposium Proceedings, Vol. 539, (Pittsburgh, Pennsylvania: Materials Research Society), p. 277, (1999)

12. K. K. Phani and S. K. Niyogi, J. Mater. Sci., 22 257, (1987)

13. E.O. Shaffer II, K.E. Howard, M.E. Mills, P.H. Townsend III, Mat. Sci. Symp. Proc., Vol 612 D1.1, 1-8, (2000)

14. X. Xiao et al., Microelectronic Engineering, 55, (2001), 53-57.

15. A.A. Volinsky, N.R. Moody, W.W. Gerberich, Acta Mater. Vol. 50/3 441, (2002)

16. G.R. Antis, P. Chantikol, B.R. Lawn and D.B. Marshall, J. of Amer. Ceram. Soc., Vol. 64, No. 9, pp. 533-538, (1981)

17. G.M. Pharr, D.S. Harding, W.C. Oliver, Mechanical Properties and Deformation Behavior of Materials Having Ultra-Fine Microstructures, M. Nastasi et al. (eds.), Kluwer Academic Press, pp. 449-461, (1993)

18. D. Harding, W. Oliver, G. Pharr, MRS Symp. Proc. Vol. 356, pp. 663-668, (1995)

19. Spectroscopic ellipsometry measurements were obtained on a commercially available JA Woollam VUV-VASE302 instrument, at three angles of incidence $\left(65\right.$ to $\left.75^{\circ}\right)$ and from 0.7 to 9.5 eV. Cf. N.V. Edwards, J. Vella, Q. Xie, S. Zollner, D. Werho, I. Adhihetty, R. Liu, T.E. Tiwald, C. Russell, J. Vires, and K.H. Junker, Materials Research Society Symposium Proceedings, Vol. 697, P4.7 (2001)

20. J.B. Vella, Q. Xie, N.V. Edwards, J. Kulik, K. Junker, Materials Research Society Symposium Proceedings, Vol. 695, L6.25 (2002)

21. A.A. Volinsky, J.B. Vella, W.W. Gerberich, submitted to Thin Solid Films (2002)

22. C.P. Ostertag, P.C. Charalambides, and A.G. Evans, Acta Mater. 37(7) 2077 (1989)

23. J.W. Hoehn, S.K. Venkataraman, H.Huang, and W.W. Gerberich, Mat. Sci and Eng. A192/193 306 (1995) 